\title{
A High Performance Architecture for Rotating Decimal Coordinates
}

\author{
Jose-Luis Sanchez, Higinio Mora, Jeronimo Mora, Antonio Jimeno \\ Computer Technology Department \\ University of Alicante \\ Alicante, Spain \\ Email: sanchez@dtic.ua.es,hmora@dtic.ua.es,jeronimo@dtic.ua.es,jimeno@dtic.ua.es
}

\begin{abstract}
Although radix-10 arithmetic has been gaining renewed importance over the last few years, high performance decimal systems and techniques are still under development. In this paper, a modification of the CORDIC method for decimal arithmetic is proposed so as to produce fast rotations. The algorithm works with BCD operands and no conversion to binary is needed. A significant reduction in the number of iterations in comparison to the original decimal CORDIC method is achieved. The experiments showing the advantages of the new method are described. Finally, the results with regard to delay obtained by means of an FPGA implementation of the method are shown.
\end{abstract}

\section{INTRODUCTION}

Numbers are commonly expressed by human beings using decimal representation; as a consequence, in the early days of computing, most of the first computers worked with decimal operands [1]. However, due to the greater simplicity of binary arithmetic unit and the compactness of binary numbers, decimal arithmetic fell into disuse and for many years it has been difficult to find new proposals of radix 10-based computers. This fact has finally led to a preponderance of binary systems over decimal ones. In spite of that, some examples of decimal architectures can be found, such as Hewlett Packard [2], Texas Instruments [3] and Casio calculators [4], and some others [4].

In recent years, a renewed interest in decimal arithmetic computing has arisen, since it is essential for many applications. For instance, financial calculations are carried out using decimal arithmetic, as binary operations often imply rounding up or down the results when working with fractional operands. Several studies involving financial and businessoriented applications have revealed that $55 \%$ of the numerical data contained in commercial databases are in decimal format [5]. The need for high precision engineering and manufacturing systems is also essential in CAD/CAM. When defining a radix10 magnitude for an object, the internal use of radix-2 usually implies loss of precision, since the equivalent binary number is likely to have an infinite amount of fractional digits. On the other hand, there are currently optic and magnetic sensors which directly provide the output in BCD format, so that the user can easily monitor the evolution of certain magnitudes and detect any errors [6]. The same happens with some types of actuators which use ISO-ASCII as the code for inputting data to the manufacturing process [7].
Proof of the importance recently given to decimal representation is the fact that even the IEEE 854 standard uses a radix-independent generalization of IEEE 754 and supports decimal floating point operations [8], [9]. Recently the IBM z900 microprocessor has been developed [10], with a decimal arithmetic unit. Furthermore, the European Commission specifies a certain number of decimal digits for calculating currency conversions [11].

CORDIC (COordinate Rotation Digital Computer) is a relevant method to approximate mathematical functions [12]. This method basically works as an iterative algorithm for approximating rotation of a two-dimensional vector using only add and shift operations. It is particularly suited to hardware implementations due to the fact that it does not require any multiplication. Originally, CORDIC was applied to binary arithmetic, but later its application was proposed for decimal data [13], [14].

This paper shows new results on the research in decimal arithmetic carried out by the authors [15]. Thus, a new CORDIC method for decimal operands is proposed, based on the use of decimal arithmetic and on the selection of adequate angles so as to reduce the number of iterations required to obtain a suitable precision. In section II, both the binary and the decimal CORDIC method are reviewed. In section III, the new CORDIC method is proposed. In order for a real system to operate with our method, an architecture carried out on FPGA is proposed throughout section IV and the results of a series of experiments with regard to precision and the required number of stages are showed. Finally, in section V, the conclusions are given.

\section{THE CORDIC METHOD}

\section{A. Reviewing the binary CORDIC method}

The rotation of a $2 \mathrm{D}$ point $(x, y)$ through an angle $\theta$ can be directly computed by means of the following equations:

$$
\begin{array}{r}
x^{\mathrm{R}}=x \cos \theta-y \sin \theta \\
y^{\mathrm{R}}=x \sin \theta+y \cos \theta
\end{array}
$$

The above equations imply a high computational cost due to the fact that some multiplications and the previous calculation of $\cos \theta$ and $\sin \theta$ must be performed. 
CORDIC was developed by Volder [12] for computing the rotation of a $2 \mathrm{D}$ vector of circular coordinates expressed as binary numbers, exclusively using addition and shift operations. Walther [16] extended the method to hyperbolic and linear coordinates. CORDIC works in two different modes. In rotation mode, a vector $\left(x_{0}, y_{0}\right)$ is rotated through an angle $\theta$ in order to obtain a new vector $\left(x_{n}, y_{n}\right)$. The overall rotation is divided into micro-rotation such that, in micro-rotation $j$, an angle $\alpha_{j}=\tan ^{-1}\left(2^{-j}\right)$ is added to or subtracted from the remaining angle $\theta_{j}$. In this way, this angle approaches zero. In vectoring mode, the vector $\left(x_{0}, y_{0}\right)$ is progressively rotated towards the $x$-axis by means of angles such as those previously mentioned, so that the component $y$ approaches 0 . As a result, the sum of all the angles applied gives the value of the angle of vector $\left(x_{0}, y_{0}\right)$ towards the $x$-axis, whereas the final component $x_{n}$ is the vector magnitude. The algorithm is based on the following equations:

$$
\begin{gathered}
x_{j+1}=x_{j}-m \sigma_{j} y_{j} 2^{-d(j)} \\
y_{j+1}=y_{j}+\sigma_{j} x_{j} 2^{-d(j)} \\
z_{j+1}=z_{j}-w_{d(j)}
\end{gathered}
$$

The values for $m$ are 1 for circular, -1 for hyperbolic, and 0 for linear coordinates. The value for $\sigma_{j}$ determines the direction of micro-rotation $j$. In rotation mode, $\sigma_{j}$ is equal to 1 if $z_{j}$ is positive, and $\sigma_{j}$ is equal to -1 otherwise. The values for $d(j)$ and $w_{d(j)}$ are shown in Table I, whereas Table II shows the results provided by the algorithm in rotation mode depending on the type of coordinates.

The elementary angles $\alpha_{j}$ must fulfil the following condition [16]:

$$
\alpha_{j} \leq \sum_{k=j+1}^{n} \alpha_{j}+\alpha_{n}, j \geq 0
$$

With regard to the elementary angles chosen for circular coordinates, convergence is guaranteed since the following property is accomplished:

$$
\tan ^{-1}\left(2^{-j}\right) \leq \sum_{k=j+1}^{n} \tan ^{-1}\left(2^{-k}\right), j \geq 0
$$

When working with hyperbolic coordinates, carrying out each micro-rotation only once is not sufficient. Indeed, convergence can be achieved by repeating certain iterations [16], as shown in Table I.

In iteration $j$, a scaling factor is added to the new coordinates $\left(x_{j}, y_{j}\right)$. This factor is given by the following expression:

$$
K_{m, j}=\sqrt{1+m 2^{-j}}
$$

The coordinates obtained after the last iteration have to be compensated by multiplying them by $K_{m}^{-1}$, taking into account that $K_{m}$ results from the following product:

$$
K_{m}=\prod_{j} K_{m, j}
$$

TABLE I

PARAMETERS FOR DIFFERENT COORDINATE TYPE

\begin{tabular}{|c|c|c|}
\hline Type & $d(j)$ & $w_{d(j)}$ \\
\hline Circular & $j$ & $\tan ^{-1}\left(2^{-j}\right)$ \\
\hline Hyperbolic & $\begin{array}{c}j-k, k \text { is the largest integer such that } \\
3^{k+1}+2 k-1 \leq 2 j\end{array}$ & $\tanh ^{-1}\left(2^{-j}\right)$ \\
\hline Linear & $j$ & $2^{-j}$ \\
\hline
\end{tabular}

TABLE II

RESULTS FOR DIFFERENT COORDINATE TYPE

\begin{tabular}{|c|c|}
\hline Type & Result \\
\hline \multirow{3}{*}{ Circular } & $x_{n}=K_{1}(x \cos z-y \sin z)$ \\
& $y_{n}=K_{1}(y \cos z+x \sin z)$ \\
$z_{n}=0$
\end{tabular}

Several methods to avoid performing the final product by $K_{m}{ }^{-1}$ and carry out the scaling compensation in parallel with each of the iterations have been proposed [17]-[20].

\section{B. Reviewing the Decimal CORDIC Method}

The CORDIC method is flexible and simple, so it is suitable for environments in which a small number of hardware resources are available. One of these environments is that of portable calculators [2]. However, these devices usually work with numbers in decimal format and, therefore, binary CORDIC cannot be directly used. In [13] and [21] the use of CORDIC for BCD operands is proposed. The modification of the method, focusing on the case of circular coordinates, is expressed by the following iterative equations:

$$
\begin{gathered}
x_{j+1}=x_{j}-\sigma_{j} y_{j} 10^{-j} \\
y_{j+1}=y_{j}+\sigma_{j} x_{j} 10^{-j} \\
z_{j+1}=z_{j}-\tan ^{-1}\left(10^{-j}\right)
\end{gathered}
$$

The drawback of this decimal CORDIC method lies on the relation between any two consecutive elementary angles in the form $\tan ^{-1}\left(10^{-j}\right)$. The relation between any two consecutive angles in the form $\tan ^{-1}\left(2^{-j}\right)$ is approximately 2 . This fact facilitates convergence in binary CORDIC, as expressed in (5). However, in the case of decimal representation, each angle is approximately 10 times smaller than the previous one, so convergence of the method cannot be directly guaranteed. This is not specific of radix 10 representation. Recall that in binary CORDIC applied to hyperbolic coordinates, certain iterations must be repeated so as to guarantee convergence. According to decimal CORDIC, each iteration but the initial one must be 
repeated 9 times so as to achieve convergence [13]. In this case, the following condition is fulfilled:

$$
\tan ^{-1}\left(10^{-j}\right) \leq 9 \sum_{k=j+1}^{n} \tan ^{-1}\left(10^{-k}\right), j \geq 0
$$

References [13] and [21] show that decimal CORDIC can compute sine and cosine functions with a 5-digit accuracy if at least 30 angular steps are performed. These results are suitable in terms of precision. However, this method cannot compete with binary CORDIC with regard of latency, since the binary method requires a smaller number of iterations so as to obtain the same precision. Therefore, the advantages of using the algorithm with $\mathrm{BCD}$ operands would be reduced to omit conversion between $\mathrm{BCD}$ and binary representation and, consequently, to avoid loss of precision.

\section{The New Decimal CORDIC Method}

The proposal for a new decimal CORDIC method is based on the selection of successive angles $\alpha_{j}$ such that:

$$
\alpha_{j}=\tan ^{-1}\left(z_{\lfloor j}\right)
$$

where $z_{\lfloor j}$ is the value resulting from truncating $z_{j}$ after the first digit on the left different from 0 . In this way, the $z$ component for accumulating the remaining angle is calculated by means of the following expression:

$$
\left.z_{j+1}=z_{j}-\tan ^{-1}\left(z_{j j}\right\rfloor\right)
$$

The angles $\alpha_{j}$ can be alternately determined in the following way:

$$
\tan \left(\alpha_{j}\right)=\tan \left(\tan ^{-1}\left(z_{\lfloor j\rfloor}\right)=z_{\lfloor j\rfloor}\right\rfloor
$$

As a consequence, the equations for the iterative computation of $x$ and $y$ are expressed as follows:

$$
\begin{gathered}
\left.x_{j+1}=x_{j}-m \sigma_{j} z_{\lfloor j}\right\rfloor y_{j} \\
y_{j+1}=y_{j}+\sigma_{j} z_{\lfloor j\rfloor} x_{j}
\end{gathered}
$$

From this point on, the new decimal CORDIC will be referred to as ND-CORDIC, whereas binary CORDIC and the previous decimal CORDIC will be referred to as B-CORDIC and D-CORDIC, respectively. Table III shows an example where the initial rotating angle is $z_{0}=0.785398$. The different values for $z_{j}, z_{\lfloor j}$, and $\tan ^{-1}\left(z_{\llcorner j}\right)$ according to each iteration $j$ are presented. Table IV shows the different values for $x_{j}$ and $y_{j}$ according to each iteration and the above mentioned rotation angle, and taking into account the initial values $x_{0}=0.931420$ and $y_{0}=0.538504$.
TABLE III

VALUES FOR $Z_{J}, \alpha_{J} \operatorname{AND~TAN}^{-1}\left(\alpha_{J}\right)$

\begin{tabular}{|c|l|l|l|}
\hline Iteration & $z_{j}$ & $z_{i j}$ & $\tan ^{-1}\left(z_{\lfloor j}\right)$ \\
\hline$j=0$ & 0.785398 & 0.7 & 0.610725 \\
\hline$j=1$ & 0.174672 & 0.1 & 0.099668 \\
\hline$j=2$ & 0.075003 & 0.07 & 0.069886 \\
\hline$j=3$ & 0.005117 & 0.005 & 0.004999 \\
\hline$j=4$ & 0.000117 & 0.0001 & 0.0001 \\
\hline$j=5$ & 0.000017 & 0.00001 & 0.00001 \\
\hline$j=6$ & 0.000007 & 0.000007 & 0.000007 \\
\hline$j=7$ & 0.000000 & 0.000000 & 0.000000 \\
\hline
\end{tabular}

TABLE IV

VALUES FOR $Z_{J}, \alpha_{J} \operatorname{AND~TAN~}^{-1}\left(\alpha_{J}\right)$

\begin{tabular}{|c|c|c|l|}
\hline Iteration & $x_{j}$ & $y_{j}$ & $\left.\alpha_{j}=z_{j}\right\rfloor$ \\
\hline$j=0$ & 0.931420 & 0.538504 & 0.7 \\
\hline$j=1$ & 0.554467 & 1.190498 & 0.1 \\
\hline$j=2$ & 0.435417 & 1.245945 & 0.07 \\
\hline$j=3$ & 0.348201 & 1.276424 & 0.005 \\
\hline$j=4$ & 0.341822 & 1.278165 & 0.0001 \\
\hline$j=5$ & 0.341694 & 1.278199 & 0.00001 \\
\hline$j=6$ & 0.341681 & 1.278202 & 0.000007 \\
\hline$j=7$ & 0.341669 & 1.278205 & 0 \\
\hline
\end{tabular}

As shown in the last row of Table IV, the final values for the rotated coordinates are $x_{7}=0.341669, y_{7}=1.278205$. The rotation of the original point, directly computed by means of (1) and (2), gives as a result the values $x^{\mathrm{R}}=0.277834$ and $y^{\mathrm{R}}=$ 1.039393. The divergence between $\left(x_{7}, y_{7}\right)$ and $\left(x^{\mathrm{R}}, y^{\mathrm{R}}\right)$ is caused by the scaling factor that is incorporated within each ND-CORDIC iteration. The computation of the factor for compensating this scaling can be obtained by means of the following expression:

$$
\left.K_{N D}{ }^{-1}=\prod_{j=0 . . n} \cos \left(\tan ^{-1}\left(z_{\lfloor j}\right\rfloor\right)\right)
$$

In Table $\mathrm{V}$, the values for the scaling compensation factor incorporated within the first iterations are shown. For relatively small values of $z_{\lfloor j\rfloor}$, the scaling factor can be assumed to be equal to 1 . The last row contains the value for the global scaling factor $K_{N D}^{-1}$ as defined in (15).

TABLE V

TERMS DETERMINING THE SCALING FACTOR

\begin{tabular}{|l|l|l|}
\hline Iteration & $\left.z_{\lfloor j}\right\rfloor$ & $\left.\cos \left(\tan ^{-1}\left(z_{\lfloor j}\right\rfloor\right)\right)$ \\
\hline$j=0$ & 0.7 & 0.81923192 \\
\hline$j=1$ & 0.1 & 0.99503719 \\
\hline$j=2$ & 0.07 & 0.99755897 \\
\hline$j=3$ & 0.005 & 0.9999875 \\
\hline$j=4$ & 0.0001 & 1 \\
\hline$j=5$ & 0.00001 & 1 \\
\hline$j=6$ & 0.000007 & 1 \\
\hline$K_{N D}{ }^{-1}$ & & 0.81316621 \\
\hline
\end{tabular}


The results of the products $x_{7} \cdot K_{N D}{ }^{-1}$ and $y_{7} \cdot K_{N D}{ }^{-1}$ give an error equal to $4.39821 \cdot 10^{-7}$ for coordinate $x$ and $1.17566 \cdot 10^{-7}$ for coordinate $y$.

The scale factor compensation by means of multiplication must be avoided due to the high computational cost of this operation. In B-CORDIC, compensation without products is easy to perform due to the fact that the scale factor is a constant [12]. However, in ND-CORDIC this factor varies depending on the different angles chosen through the method iterations, as shown in Table V.

A technique based on LUT (look-up tables) can be used which allows the compensation to be performed on each iteration. Equations (13) and (14) can be modified in order to include the compensation, which results in the following expression, where the superscript $C$ indicates that the coordinates are scaling-compensated:

$$
\begin{gathered}
x_{j+1}{ }^{C}=\left(x_{j}-m \sigma_{j} z_{\lfloor j\rfloor} y_{j}\right) \cos \left(\tan ^{-1}\left(z_{\lfloor j\rfloor}\right)\right) \\
y_{j+1}{ }^{C}=\left(y_{j}+\sigma_{j} z_{\lfloor j\rfloor} x_{j}\right) \cos \left(\tan ^{-1}\left(z_{\lfloor j\rfloor}\right)\right)
\end{gathered}
$$

The above equations can be rewritten in the following way:

$$
\begin{gathered}
x_{j+1}{ }^{C}=x_{j} \cos \left(\tan ^{-1}\left(z_{\lfloor j\rfloor}\right)-m \sigma_{j} z_{\lfloor j\rfloor} y_{j} \cos \left(\tan ^{-1}\left(z_{\lfloor j\rfloor}\right)\right)\right. \\
\left.y_{j+1}{ }^{C}=y_{j} \cos \left(\tan ^{-1}\left(z_{\lfloor j}\right\rfloor\right)+\sigma_{j} z_{\lfloor j\rfloor} x_{j} \cos \left(\tan ^{-1}\left(z_{\lfloor j\rfloor}\right\rfloor\right)\right)
\end{gathered}
$$

In equations (18) and (19), four different terms appear:

$$
\begin{gathered}
t_{x, 0}=x_{j} \cos \left(\tan ^{-1}\left(z_{\lfloor j\rfloor}\right)\right) \\
t_{y, 0}=y_{j} \cos \left(\tan ^{-1}\left(z_{\lfloor j\rfloor}\right\rfloor\right) \\
\left.t_{x, 1}=z_{\lfloor j\rfloor} y_{j} \cos \left(\tan ^{-1}\left(z_{\lfloor j\rfloor}\right\rfloor\right)\right) \\
\left.t_{y, 1}=z_{\lfloor j\rfloor} x_{j} \cos \left(\tan ^{-1}\left(z_{\lfloor j}\right\rfloor\right)\right)
\end{gathered}
$$

The proposed compensation technique consists in storing the above four terms in four independent blocks of LUT. The entries for each block of LUT consist of the one-digit mantissa and the exponent of $z_{\lfloor j}$, and also the value of $x_{j}$ or $y_{j}$. If each term was stored on a single LUT, the size of each LUT would be excessive. For instance, when a precision of 6 fractional digits is required, 24 bits are needed for each coordinate, 4 for indicating the mantissa of $\mathrm{z}_{L_{j}}$, and 3 for indicating the exponent of $z_{\lfloor j\rfloor}$ (from 000 to 110). Thus, the size of a monolithic LUT for each term would be $2^{4 \cdot 6+3+4} \cdot 4 \cdot 6=6144 \mathrm{MB}$. Instead, much smaller LUT can be used. If the different BCD X3 digits of $x_{j}$ are considered, the term $t_{x 0}$ can be expressed as:

$$
\left.t_{x, 0}=\left(x_{j}[5] x_{j}[4] x_{j}[3] x_{j}[2] x_{j}[1] x_{j}[0]\right) \cos \left(\tan ^{-1}\left(z_{j}\right\rfloor\right)\right)
$$

Therefore, each small LUT will receive as inputs the value of a single digit of the coordinate and the mantissa and exponent of $z_{j}$. For 6 fractional digits, the size of each LUT would be $2^{4+3+4} \cdot 4 \cdot 6=6 \mathrm{~KB}$. Since 6 fractional digits and four terms must be considered, the overall memory size would be $6 \mathrm{~KB} \cdot 6 \cdot 4=144 \mathrm{~KB}$. In this case, $6 \mathrm{LUT}$ would constitute the storage block for $t_{x, 0}$, other 6 LUT would compound the LUT block for $t_{x, 1}$, and so

Therefore, each small LUT will receive as inputs the value of a single digit of the coordinate and the mantissa and exponent of $z_{\lfloor j\rfloor}$. For 6 fractional digits, the size of each LUT would be $2^{4+3+4} \cdot 4 \cdot 6=6 \mathrm{~KB}$. Since 6 fractional digits and four terms must be considered, the overall memory size would be $6 \mathrm{~KB} \cdot 6 \cdot 4=144 \mathrm{~KB}$. In this case, $6 \mathrm{LUT}$ would constitute the storage block for $t_{x, 0}$, other 6 LUT would compound the LUT block for $t_{x, 1}$, and so on.

\section{DeCIMAL CORDIC ARCHITECTURE. EXPERIMENTATION}

\section{A. Some Details on the Architecture Implementation}

Addition on BCD operands is more complex than binary addition since the carry resulting from the sum of two digits must be propagated to the sum of the following ones [13]. Moreover, the sum of two BCD digits must be corrected adding the value 6 to this sum if it is greater than 9 .

BCD X3 representation allows decimal addition/subtraction to be more efficiently performed, since only two 4-bit binary adders are required for each pair of digits. The final result is directly obtained in $\mathrm{BCD}$ X3. More detailed information on BCD X3 adders can be found in [13]. Conversion from BCD to $\mathrm{BCD}$ X3 requires only 10 gates distributed over 3 level, and similar resources are needed when transforming $\mathrm{BCD} X 3$ into BCD operands. Therefore, the use of BCD X3 is proposed since addition, subtraction, and other operations are simpler than for BCD.

The complete architecture for each of the iterations of the proposed ND-CORDIC method is shown in Fig 1.

\section{B. Experiments on Precision}

Different tests were carried out so as to make a complete comparison with regard to precision between B-CORDIC, DCORDIC, and the ND-CORDIC method proposed in this work. Values within the range $[0,1)$ were chosen for the $(x, y)$ coordinates and also for the rotation angle $\theta$. Original data were represented in $\mathrm{BCD}$ with 6 fractional digits. For DCORDIC and ND-CORDIC, a conversion stage from BCD to BCD X3 was included, whereas for B-CORDIC the BCD operands were converted into binary numbers. In any case, 28bit operands were considered. The experiments were aimed at comparing the number of iterations required in each method so as to achieve suitable precision. In the test, 500 random points were rotated through a random angle. The results for the error distribution are shown in Fig. 2, whereas the results for the maximum relative error are shown in Fig. 3. 


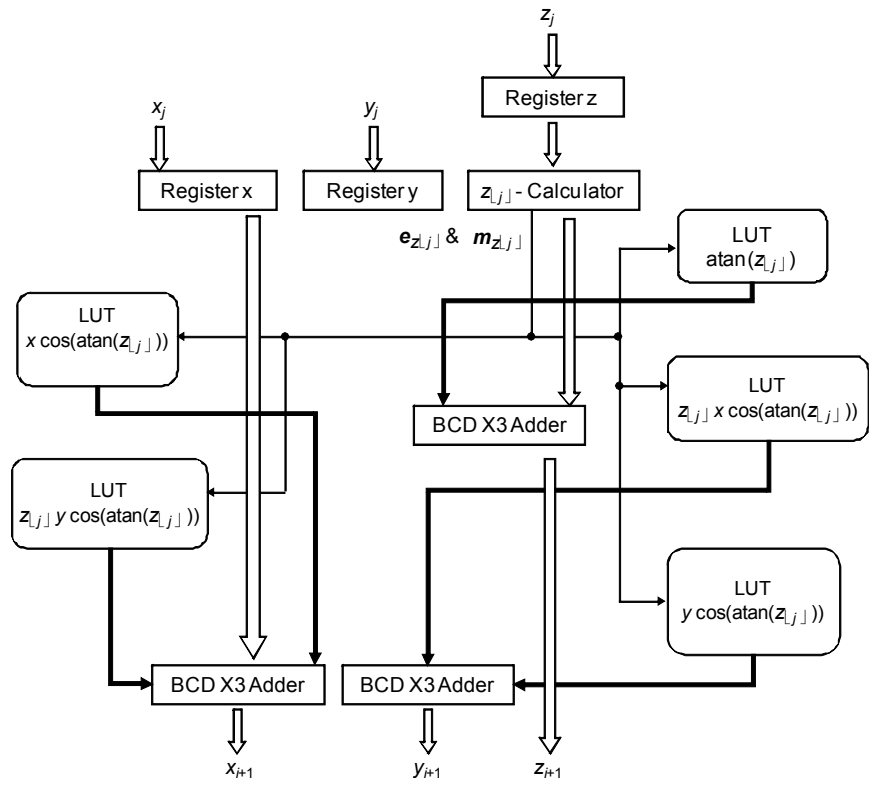

Fig. 1. The architecture for an ND-CORDIC iteration.

A decreasing tendency can be observed for every method as the number of iterations increases. However, the error decreases much faster for ND-CORDIC. For this method, the error reaches stability in about 10 iterations, whereas for DCORDIC and B-CORDIC much more iterations are required. In addition, the maximum error is always lower for the NDCORDIC method. The mean and maximum relative error for ND-CORDIC seems to be always lower than those for DCORDIC and B-CORDIC.

\section{Experiments on Latency and Hardware resources}

The proposed architecture was implemented on VHDL using the Xilinx ISE 7.1i tool. The Virtex4 XC4VLX60 FPGA was chosen for simulation. The architectures for D-CORDIC proposed in [13] and [21] and for B-CORDIC were also implemented. In all cases, a complete stage of the method was implemented, with the type of adder and shifter, if needed, being varied according to each method. The global method was implemented on an unfolded architecture. Conventional arithmetic was used. In case of B-CORDIC, the scaling factor was compensated by means of the method proposed in [20], which allows the compensating product to be transformed into simple additional shift-add iterations. In case of ND-CORDIC, the compensation was achieved by means of the LUT technique previously described. The initial conversion from $\mathrm{BCD}$ to $\mathrm{BCD} \mathrm{X} 3$ and the final conversion the other way were also included for the D-CORDIC and the ND-CORDIC methods. For B-CORDIC, an initial conversion from BCD to binary and a final conversion the other way were also implemented. A homogeneous length of 28 bits was used for every number format, so six fractional digits, corresponding to 24 bits, were considered for the BCD original numbers.

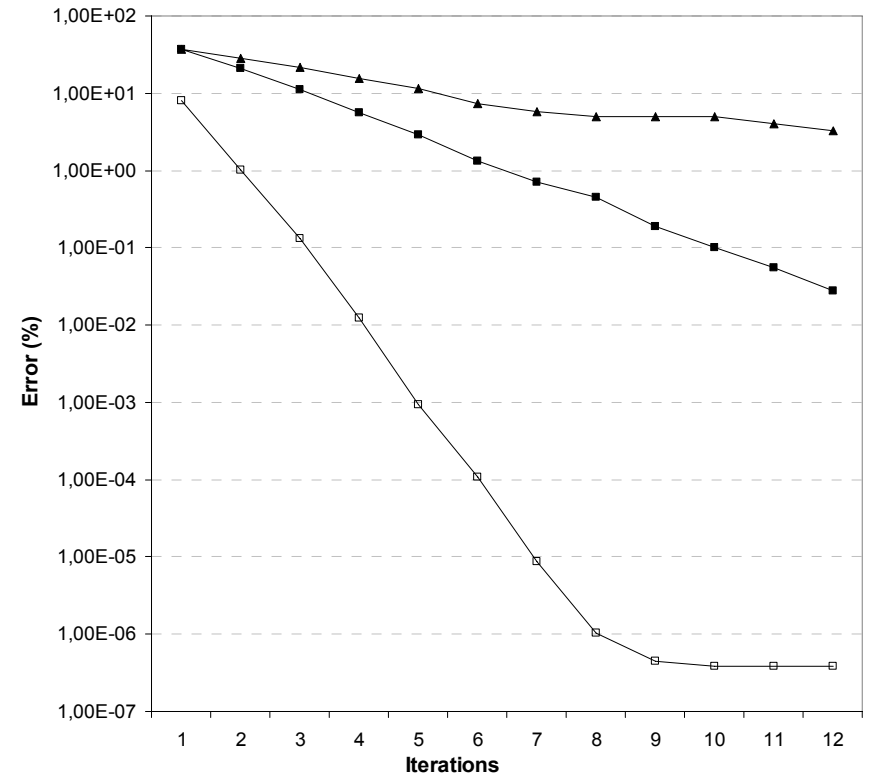

Fig. 2. Relative error distribution when calculating the rotation of vectors within the circumference unit, according to the number of iterations; logarithmic scale ( $\boldsymbol{\Delta}=$ D-CORDIC; $\mathbf{\square}=$ B-CORDIC; $\square=$ ND-CORDIC)

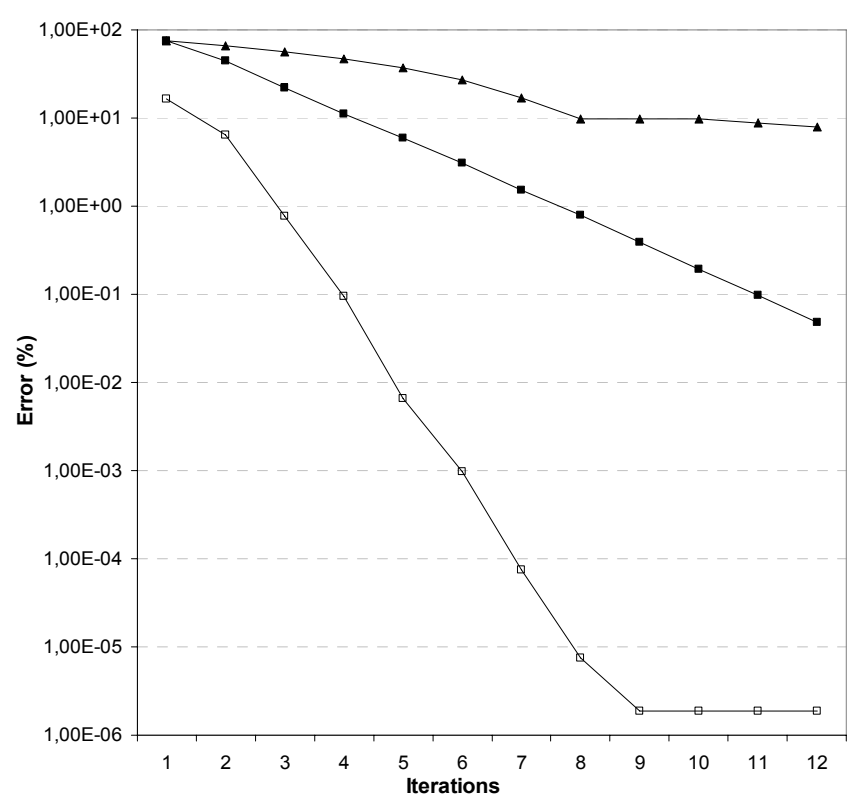

Fig. 3. Maximum relative error on calculating the rotation of vectors within the circumference unit, according to the number of iterations; logarithmic scale ( $\boldsymbol{\Delta}=$ D-CORDIC; $\boldsymbol{\square}=$ B- CORDIC; $\square=$ ND-CORDIC).

The results for the delays and the FPGA resources used, when considering a single iteration and including number format conversions and scaling compensation, are shown in Table VI for comparison. As it can be observed, the delay for the proposed ND-CORDIC is less than half the delay for the 
conventional D-CORDIC. Although B-CORDIC has the least delay per iteration, it must be taken into account that a higher number of iterations must be performed so as to achieve a precision similar to the one obtained with ND-CORDIC.

An overall comparison between the three methods including precision and delay, has been performed. The results are shown in Fig. 4, where the product of the mean relative error and the delay in nanoseconds is depicted for each architecture. It can be observed that ND-CORDIC offers better global performance than D-CORDIC and B-CORDIC for the considered iterations.

TABLE VI

SiNGLE Stage DELAY FOR DifFERENT CORDIC ARCHITECTURES

\begin{tabular}{|l|c|c|c|}
\hline Results & B-CORDIC & D-CORDIC & ND-CORDIC \\
\hline Delay (ns) & 48.413 & 225.182 & 103.042 \\
\hline Slices & $909(3 \%)$ & $5785(21 \%)$ & $8585(32 \%)$ \\
\hline 4-input LUT & $1626(3 \%)$ & $5416(10 \%)$ & $9746(18 \%)$ \\
\hline Bonded IOB & $172(26 \%)$ & $172(26 \%)$ & $172(26 \%)$ \\
\hline
\end{tabular}

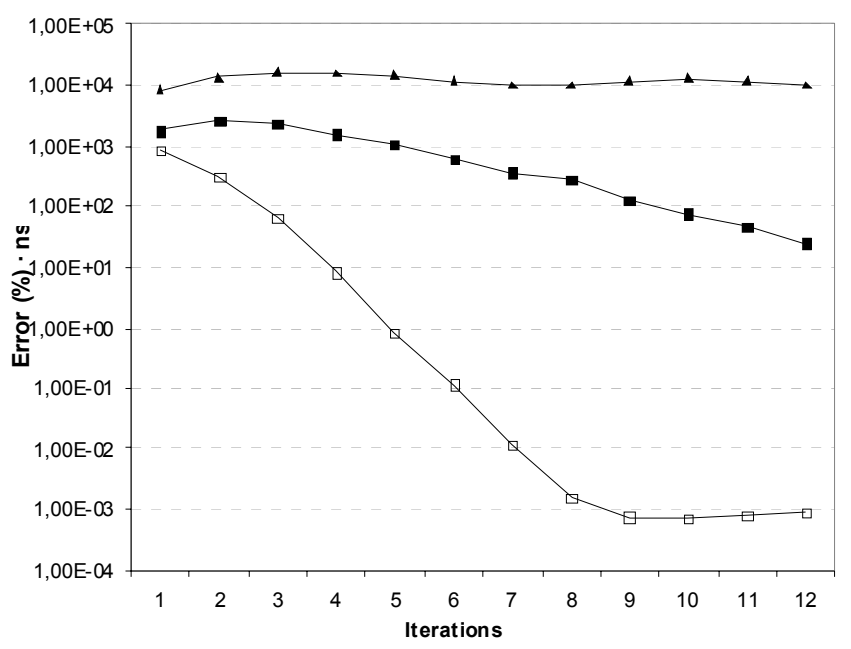

Fig. 4. Mean relative error $\times$ delay for each CORDIC architecture; logarithmic scale ( $\boldsymbol{\Lambda}=$ D-CORDIC; $\boldsymbol{\square}=$ B- CORDIC; $\square$ = ND-CORDIC ).

\section{CONCLUSIONS}

One of the most important tasks in new hardware design is to achieve high performance rates with a trade-off between precision and delays of the circuitry that forms these new embedded architectures. It seems that there is a growing trend towards developing new systems integrating decimal arithmetic, which is required in many practical research areas.

In this work a new CORDIC method for performing rotations on decimal coordinates has been proposed. The tests performed confirm that the proposed method requires fewer number of iterations so as to obtain a required precision. Moreover, the maximum error obtained is always lower for the proposed method than for binary and decimal CORDIC.
On the other hand, with regard to latency, the experiments show that the proposed method has a much lower delay than that for decimal CORDIC. In addition, the indices obtained are not very far from those obtained by binary CORDIC.

As a future work, an interesting task consists in developing a hardware implementation of a specific CORDIC-based rotator embedded on a decimal architecture. At this point, new scaling compensation techniques must be studied and developed so as to improve delay and resources utilization.

This work has been supported by the Generalitat Valenciana (Spain) under Grant No. GV/2007/173.

\section{REFERENCES}

[1] H. H. Goldstine and A. Goldstine, "The Electronic Numerical Integrator and Computer (ENIAC)," IEEE Annals Hist. Comput., vol. 18 \#1, pp. 10$16,1996$.

[2] D. S. Cochran. "Algorithms and Accuracy in the HP-35," HP Journal, pp. 10-11, July 1972.

[3] TI-89/TI-92 Plus Developers Guide, Beta Version .02, Texas Instruments, Jan. 2001.

[4] M. F. Cowlishaw, "Decimal Floating Point: Algorism for Computers," Proc. 16th IEEE Symp. Computer Arithmetic, 2003.

[5] A. Tsang and M. Olschanowsky, "A Study of Database 2 Customer Queries," IBM Santa Teresa Laboratory, San Jose, CA, Technical Report TR-03-413, 1991.

[6] S. Kim, J. Kwon, S. Kim, and B. Lee, "Multiplexed Strain Sensor using Fiber Gratin-Tuned Fiber Laser with a Semiconductor Optical Amplifier," IEEE Photonics Technology Letters, vol. 13, no. 4, pp. 350351, 2001 .

[7] S. McMains, J. Smith, and C. Séquin, "The evolution of a layered manufacturing interchange format," Proc. DETC02, ASME Design Engineering Technical Conferences, pp.945-953, Sept. 2002.

[8] IEEE 854-1987 - IEEE Standard for Radix-Independent Floating-Point Arithmetic, The Institute of Electrical and Electronics Engineers, Inc., New York, 1987.

[9] Draft IEEE Standard for Floating-Point Arithmetic, The Institute of Electrical and Electronics Engineers, Inc., New York, 2005.

[10] F. Y. Busaba, C. A. Krygowski, W. H. Li, E. M. Schwarz, and S. R. Carlough, "The IBM z900 Decimal Arithmetic Unit," Proc.35th Asilomar Conf. Signals, Systems and Computers, pp. 1335-1339, 2001.

[11] European Commission Directorate General II, "The Introduction of the Euro and the Rounding of Currency Amounts," Note II/28/99-EN Euro Papers no. 22., 32pp, Belgium, 1999.

[12] J. Volder, "The CORDIC Trigonometric Computing Technique," IRE Trans. Electron. Comput., vol. EC-8, no. 3, pp. 330-334, 1959.

[13] H. Schmid, Decimal Computation. NY: John Wiley \& Sons, 1974.

[14] J. C. Kropa, "Calculator Algorithms," Mathematics Magazine, vol. 51, no. 2, pp. 106-109, March 1978.

[15] J. -L. Sanchez, A. Jimeno, H. Mora, J. Mora, and F. Pujol, "A CORDICbased Architecture for High Performance Decimal Calculations," Proc. 2007 International Symposium on Industrial Electronics, pp. 1951-1956, Jun. 2007.

[16] J. S. Walther, "A unified algorithm for elementary functions," Proc. AFIPS Spring Joint Computer Conf., 1971, pp. 379-385.

[17] A. Despain, "Fourier Transform Computers Using CORDIC Iterations," IEEE Trans. Comput., vol. C-23, no. 10, pp. 993-1001, Oct. 1974.

[18] E. F. Deprettere, P. Dewilde, and R. Udo, "Pipelined CORDIC architecture for fast VLSI filtering and array processing," Proc. ICASSP'84, 1984, pp. 41.A.6.1-41.A.6.4.

[19] G. Haviland and A. Tuszynski, "A CORDIC Arithmetic Processor Chip," IEEE Trans. Comput., vol. C-29, no. 2, pp. 68-79, Feb. 1990.

[20] D. Timmermann, H. Hahn, B. J. Hosticka, and B. Rix, "A new addition scheme and fast scaling factor compensation methods for CORDIC algorithms," INTEGRATION, the VLSI Journal, 11, pp. 85-100, 1991.

[21] H. Schmid and A. Bogacki, "Use decimal CORDIC for generation of many transcendental functions," EDN, pp. 64-73, Feb. 1973. 